Proceedings of the Edinburgh Mathematical Society (2004) 47, 495-512 (C)

DOI:10.1017/S0013091503000294 Printed in the United Kingdom

\title{
MULTIPLICITY OF POSITIVE SOLUTIONS UNDER NONLINEAR BOUNDARY CONDITIONS FOR DIFFUSIVE LOGISTIC EQUATIONS
}

\author{
KENICHIRO UMEZU \\ Faculty of Engineering, Maebashi Institute of Technology, \\ Maebashi 371-0816, Japan (ken@maebashi-it.ac.jp)
}

(Received 14 April 2003)

\begin{abstract}
In this paper we consider the existence and multiplicity of positive solutions of a nonlinear elliptic boundary-value problem with nonlinear boundary conditions which arises in population dynamics. While bifurcation problems from lines of trivial solutions are studied, the existence of bifurcation positive solutions from infinity is discussed. The former will be caught by the reduction to a bifurcation equation following the Lyapunov and Schmidt procedure. The latter will be based on a variational argument depending on the corresponding constrained minimization problem.
\end{abstract}

Keywords: bifurcation equations; secondary bifurcation; constrained minimization problems; positive solutions; multiplicity results; population dynamics

2000 Mathematics subject classification: Primary 35J65; 35J20; 35P30; 35B32; 92D25

\section{Introduction and main results}

In this paper we consider the following nonlinear elliptic eigenvalue problem:

$$
\left.\begin{array}{rlrl}
-\Delta u & =\lambda(m(x)-u) u & & \text { in } D, \\
\frac{\partial u}{\partial \boldsymbol{n}} & =\lambda b(x) g(u) & & \text { on } \partial D .
\end{array}\right\}
$$

Here $D$ is a bounded domain of $\mathbb{R}^{N}, N \geqslant 2$, with smooth boundary $\partial D, \lambda$ is a positive parameter, $m \in C^{\theta}(\bar{D})$ is a sign-changing, Hölder-continuous function with exponent $0<$ $\theta<1$ on the closure $\bar{D}, b \in C^{1+\theta}(\partial D)$ satisfies $b \geqslant 0$ and $b \not \equiv 0$ on $\partial D, g \in C^{1+\theta}([0, \delta])$ for any $\delta>0$, and $\boldsymbol{n}$ is the unit exterior normal to $\partial D$.

It is well known (cf. $[\mathbf{5}, \mathbf{6}]$ ) that problem (1.1) originates from population dynamics. Here the unknown function $u$ denotes the steady state of the density of a population diffusing at rate $1 / \lambda$, and $m(x)$ represents the growth or decay rate. Our boundary condition of the form above may suggest, from an ecological point of view, that if the species in the interior of $D$ walks toward and reaches the boundary $\partial D$, then it returns to the interior with some reaction according to $b(x) g(u)$.

If $u \in C^{2}(\bar{D})$ is a solution of (1.1) for some $\lambda>0$, then we call a pair $(\lambda, u)$ a solution of (1.1). A solution $(\lambda, u)$ of (1.1) is called positive if $u>0$ in $D$. 
Our fundamental assumption for $g$ is that

$$
g(0)=g^{\prime}(0)=0 .
$$

Then any positive solution $(\lambda, u)$ of (1.1) satisfies $u>0$ in $\bar{D}$ by using the strong maximum principle and the boundary point lemma (cf. [12]). Moreover, problem (1.1) has two trivial solution lines; one is $\{(\lambda, 0)\}$, and the other is $\{(0, c): c$ is a constant $\}$ (simply $\{(0, c)\})$, which bifurcates from $\{(\lambda, 0)\}$ at $(\lambda, u)=(0,0)$.

First we study bifurcation of positive solutions from $\{(\lambda, 0)\}$ for (1.1). Let $\gamma_{1}(\lambda)$ be a unique principal eigenvalue of the linearized eigenvalue problem

$$
\left.\begin{array}{rlrl}
-\Delta \varphi & =\lambda m(x) \varphi+\gamma(\lambda) \varphi & & \text { in } D, \\
\frac{\partial \varphi}{\partial \boldsymbol{n}} & =0 & & \text { on } \partial D .
\end{array}\right\}
$$

Here a principal eigenvalue is characterized by having a positive eigenfunction. When $\int_{D} m \mathrm{~d} x<0$, there exists a unique positive number $\lambda_{1}(m)$ such that $\gamma_{1}\left(\lambda_{1}(m)\right)=0$, whereas $\gamma_{1}(\lambda)<0$ for all $\lambda>0$ when $\int_{D} m \mathrm{~d} x \geqslant 0$ (see [1]). In fact, if $(\lambda, 0)$ is a bifurcation point for (1.1), then $\gamma_{1}(\lambda)=0$ (see [2]), that is, $\lambda=0, \lambda_{1}(m)$. Here it is understood that $\lambda_{1}(m)=0$ if $\int_{D} m \mathrm{~d} x \geqslant 0$.

In the linearized case of our boundary condition at $u=0$, that is, in the Neumann case $\partial u / \partial \boldsymbol{n}=0$, it is well known (cf. [2]) that problem (1.1) has a unique positive solution $u_{\lambda}$ for every $\lambda>\lambda_{1}(m)$ and no positive solution for any $0<\lambda \leqslant \lambda_{1}(m)$. When $\int_{D} m \mathrm{~d} x \geqslant 0$, Hess [8] characterizes the limiting behaviour of the unique positive solution $u_{\lambda}$ as $\lambda \downarrow 0$ by the assertion that $u_{\lambda} \rightarrow \int_{D} m \mathrm{~d} x /|D|$ in $C(\bar{D})$ as $\lambda \downarrow 0$, where $|D|$ denotes the volume of $D$. When $\int_{D} m \mathrm{~d} x<0$, it can be verified by use of the local bifurcation theory due to Crandall and Rabinowitz [7] that the bifurcation of positive solutions can occur to the right at $\left(\lambda_{1}(m), 0\right)$.

Also, in case (1.2) it is easy to check that the local bifurcation theory is applicable both at $(\lambda, u)=(0,0)$ and at $\left(\lambda_{1}(m), 0\right)$ whenever $\int_{D} m \mathrm{~d} x \neq 0$. In fact, by applying it especially at $(\lambda, u)=(0,0)$, we can show that if $(\lambda, u)$ is a solution of $(1.1)$ near $(\lambda, u)=$ $(0,0)$, then $(\lambda, u)$ is on $\{(\lambda, 0)\} \cup\{(0, c)\}$. For this reason we restrict our consideration to the case $\int_{D} m \mathrm{~d} x=0$.

Since $\lambda \leqslant 0$ is outside the scope of this paper, we call $(\lambda, u)=(0,0)$ a bifurcation point to the right for (1.1) if there exist positive solutions $\left(\lambda_{j}, u_{\lambda_{j}}\right)$ of (1.1) that satisfy $\lambda_{j} \downarrow 0$ and $u_{\lambda_{j}} \rightarrow 0$ in $C(\bar{D})$ as $j \rightarrow \infty$.

Now the following result gives a complete description in the analytic case of $g$ for the possibility that $(\lambda, u)=(0,0)$ is a bifurcation point to the right.

Theorem 1.1. Suppose $\int_{D} m \mathrm{~d} x=0$, and suppose $g$ is analytic at $u=0$ with $g(0)=$ $g^{\prime}(0)=0$. If either

(a)

$$
g^{\prime \prime}(0)<\frac{2|D|}{\int_{\partial D} b \mathrm{~d} \sigma}
$$

or 
(b)

$$
g^{\prime \prime}(0)=\frac{2|D|}{\int_{\partial D} b \mathrm{~d} \sigma},
$$

0 is a zero of $g(u)-\left(|D| / \int_{\partial D} b \mathrm{~d} \sigma\right) u^{2}$ of order $k_{0} \geqslant 3$, and $g^{\left(k_{0}\right)}(0)<0$,

then $(\lambda, u)=(0,0)$ is a bifurcation point to the right for (1.1). Otherwise, this is not the case.

Remark 1.2. Indeed, the assertion of Theorem 1.1 that $(\lambda, u)=(0,0)$ is a bifurcation point to the right for (1.1) implies that there exist a constant $\varepsilon>0$ and analytic functions $\lambda(\cdot):(-\varepsilon, \varepsilon) \rightarrow \mathbb{R}, v_{1}(\cdot):(-\varepsilon, \varepsilon) \rightarrow X$, where $X=\left\{v \in C^{2+\theta}(\bar{D}): \int_{D} v \mathrm{~d} x=0\right\}$, satisfying

$$
\begin{aligned}
\lambda(0) & =0 \\
\lambda(\alpha) & >0 \quad \text { for } 0<\alpha<\varepsilon, \\
v_{1}(0) & =0
\end{aligned}
$$

such that $(\lambda, u)=\left(\lambda(\alpha), \alpha\left(1+v_{1}(\alpha)\right)\right)$ is a positive solution of (1.1) for each $0<\alpha<\varepsilon$. Moreover, if $(\lambda, u)$ is a positive solution of (1.1) for $\lambda>0$ and $\|u\|_{C^{2+\theta}(\bar{D})}$ both small enough, then $(\lambda, u)=\left(\lambda(\alpha), \alpha\left(1+v_{1}(\alpha)\right)\right)$ for some $0<\alpha<\varepsilon$ (cf. [7, Theorem 1.7] and $[\mathbf{9}$, Theorem 2.2.1]).

The proof of Theorem 1.1 will be carried out by the reduction of (1.1) to a bifurcation equation in $\mathbb{R}^{2}$, following the Lyapunov and Schmidt procedure, and by the analysis of the bifurcation equation around $(\lambda, u)=(0,0)$ by means of the implicit function theorem and the Morse lemma.

Now, the following result is a generalization of Theorem 1.1 to the Hölder class of $g$.

Corollary 1.3. Suppose $\int_{D} m \mathrm{~d} x=0$, and suppose for some $\delta>0$ that $g \in C^{1+\theta}([0, \delta])$ is non-negative and $g(0)=g^{\prime}(0)=0$. If

$$
\limsup _{u \downarrow 0} \frac{g(u)}{u^{2}}<\frac{|D|}{\int_{\partial D} b \mathrm{~d} \sigma} \quad\left(\text { respectively, } \frac{|D|}{\int_{\partial D} b \mathrm{~d} \sigma}<\liminf _{u \downarrow 0} \frac{g(u)}{u^{2}} \leqslant \infty\right),
$$

then $(\lambda, u)=(0,0)$ is (respectively, is not) a bifurcation point to the right for (1.1). Here, $\mathrm{d} \sigma$ is the surface element of $\partial D$. In a critical case such as

$$
g(u)=\frac{|D|}{\int_{\partial D} b \mathrm{~d} \sigma} u^{2}+h(u), \quad \text { where } \lim _{u \downarrow 0} \frac{h(u)}{u^{2}}=0,
$$

$(\lambda, u)=(0,0)$ is (respectively, is not) a bifurcation point to the right for (1.1) whenever we can take some integer $k \geqslant 3$ such that

$$
-\infty \leqslant \limsup _{u \downarrow 0} \frac{h(u)}{u^{k}}<0 \quad\left(\text { respectively, } 0<\liminf _{u \downarrow 0} \frac{h(u)}{u^{k}} \leqslant \infty\right) .
$$


Remark 1.4. Indeed, the assertion of Corollary 1.3 that $(\lambda, u)=(0,0)$ is a bifurcation point to the right for (1.1) implies that there exists a positive solution $\left(\lambda, u_{\lambda}\right)$ of $(1.1)$ for any $\lambda>0$ small (simply for $0<\lambda \ll 1$ ) and $u_{\lambda} \rightarrow 0$ as $\lambda \downarrow 0$ in $C^{2+\theta}(\bar{D})$ by the standard elliptic regularity.

For nonlinearity of the form $g(u)=u^{p}, p>1$, Corollary 1.3 can tell us exactly about the possibility of the bifurcation to the right at $(\lambda, u)=(0,0)$, as follows.

Example 1.5. Put $1<p<q, c_{1}>0, c_{2} \in \mathbb{R}$, and $g(u)=c_{1} u^{p}+c_{2} u^{q}$. The origin $(\lambda, u)=(0,0)$ is a bifurcation point to the right for (1.1) either if $p>2$, if $p=2$ and $c_{1}<|D| / \int_{\partial D} b \mathrm{~d} \sigma$, or if $p=2, c_{1}=|D| / \int_{\partial D} b \mathrm{~d} \sigma$ and $c_{2}<0$. Otherwise, this is not the case.

Corollary 1.3 is proved by the method of super and subsolutions, based on the results in the analytic case. Here the hypothesis that $g$ is non-negative for $u>0$ small must be used.

Next we study secondary bifurcation of positive solutions from the line $\{(0, c)\}$ for (1.1), where $g(u)=u^{p}, p>1$ :

$$
\left.\begin{array}{rlrl}
-\Delta u & =\lambda(m(x)-u) u & & \text { in } D, \\
\frac{\partial u}{\partial \boldsymbol{n}} & =\lambda b(x) u^{p} & & \text { on } \partial D .
\end{array}\right\}
$$

For a constant $c>0$ we call $(0, c)$ a secondary bifurcation point for (1.8) if there exist positive solutions $\left(\lambda_{j}, u_{\lambda_{j}}\right)$ of (1.8) that satisfy $\lambda_{j} \downarrow 0$ and $u_{\lambda_{j}} \rightarrow c$ in $C(\bar{D})$ as $j \rightarrow \infty$. To characterize secondary bifurcation points for (1.8) it is necessary to consider zeros of the function

$$
\phi(t)=\int_{D} m \mathrm{~d} x-t|D|+t^{p-1} \int_{\partial D} b \mathrm{~d} \sigma, \quad t>0,
$$

see $(4.1)$ in $\S 4$.

Now we state the following theorem.

\section{Theorem 1.6.}

(I) Assume that $\int_{D} m \mathrm{~d} x>0$. Either if $1<p<2$, or if $p=2$ and $|D|>\int_{\partial D} b \mathrm{~d} \sigma$, then problem (1.8) has a unique secondary bifurcation point $\left(0, c_{1}\right)$, where $c_{1}$ is a unique zero of $\phi$. Either if $p=2$ and $|D| \leqslant \int_{\partial D} b \mathrm{~d} \sigma$, or if $p>2$ and $|D|<m_{p} \int_{\partial D} b \mathrm{~d} \sigma$, then there is no secondary bifurcation point for (1.8). Here $m_{p}$ is a positive constant given by

$$
m_{p}=(p-1)^{p-1}\left(\frac{\bar{m}}{p-2}\right)^{p-2}
$$

where $\bar{m}=\int_{D} m \mathrm{~d} x /|D|$. Finally, if $p>2$ and $|D|>m_{p} \int_{\partial D} b \mathrm{~d} \sigma$, then problem (1.8) has exactly two secondary bifurcation points $\left(0, c_{1}\right)$ and $\left(0, c_{2}\right), c_{1}<c_{2}$, where $c_{1}$ and $c_{2}$ are two zeros of $\phi$.

(II) Assume that $\int_{D} m \mathrm{~d} x<0$. Either if $p>2$, or if $p=2$ and $|D|<\int_{\partial D} b \mathrm{~d} \sigma$, then problem (1.8) has a unique secondary bifurcation point $\left(0, c_{1}\right)$, where $c_{1}$ is a unique 
zero of $\phi$. Either if $p=2$ and $|D| \geqslant \int_{\partial D} b \mathrm{~d} \sigma$, or if $1<p<2$ and $|D|>m_{p} \int_{\partial D} b \mathrm{~d} \sigma$, then there is no secondary bifurcation point for (1.8). Finally, if $1<p<2$ and $|D|<m_{p} \int_{\partial D} b \mathrm{~d} \sigma$, then problem (1.8) has exactly two secondary bifurcation points $\left(0, c_{1}\right)$ and $\left(0, c_{2}\right), c_{1}<c_{2}$, where $c_{1}$ and $c_{2}$ are two zeros of $\phi$.

(III) Assume that $\int_{D} m \mathrm{~d} x=0$. If $p \neq 2$, then problem (1.8) has a unique secondary bifurcation point $\left(0, c_{1}\right)$, where $c_{1}$ is a unique zero of $\phi$, whereas there is no secondary bifurcation point for (1.8) if $p=2$ and $|D| \neq \int_{\partial D} b \mathrm{~d} \sigma$.

\section{Remark 1.7.}

(1) Indeed, for $\left(0, c_{j}\right), j=1,2$, respectively, of Theorem 1.6 it will be verified that there exist a constant $\bar{\lambda}_{j}>0$ and a continuous function $u(\cdot):\left[0, \bar{\lambda}_{j}\right) \rightarrow C^{2+\theta}(\bar{D})$ satisfying $u(0)=c_{j}$, such that $(\lambda, u)=(\lambda, u(\lambda))$ is a positive solution of (1.8) for each $0<\lambda<\bar{\lambda}_{j}$. Moreover, if $(\lambda, u), \lambda>0$, is a positive solution of (1.8) near $(\lambda, u)=\left(0, c_{j}\right)$, then $(\lambda, u)=(\lambda, u(\lambda))$ for some $0<\lambda<\bar{\lambda}_{j}$.

(2) If $\int_{D} m \mathrm{~d} x=0, p=2$ and $|D|=\int_{\partial D} b \mathrm{~d} \sigma$, then some positive answers to the secondary bifurcation problem can be obtained by adding certain assumptions. However, the answers seem to be implicit or not plain, which is the reason why this case is not mentioned.

The critical value $|D| / \int_{\partial D} b \mathrm{~d} \sigma$ appearing in Corollary 1.3 (cf. Example 1.5) seems to be interesting from an ecological point of view. To explain this, we consider (1.8) with the condition that $N=2, p=2, \int_{D} m \mathrm{~d} x=0$ and $b \equiv 1$. We can then show that problem (1.8) has a minimal positive solution $\left(\lambda, \underline{u}_{\lambda}\right)$ if there exists a positive solution. This is based on the construction of subsolutions that are small enough (see Lemma 3.3). So, Theorems 1.1 and 1.6 characterize the limiting behaviour of the minimal positive solution $\left(\lambda, \underline{u}_{\lambda}\right)$ as $\lambda \downarrow 0$. Indeed, let $\nu_{D}=|D| /|\partial D|$ be the ratio of the area of $D$ to the length of its boundary $\partial D$. As $\lambda \downarrow 0$, Theorems 1.1 and 1.6 assert that $\underline{u}_{\lambda} \rightarrow 0$ if $\nu_{D}>1$, that $\underline{u}_{\lambda} \rightarrow c_{1}$ if we add some certain assumptions to the condition $\nu_{D}=1$, as mentioned in Remark $1.7(2)$, and that there is no minimal positive solution $\left(\lambda, \underline{u}_{\lambda}\right)$ that is bounded in $0<\lambda \ll 1$ if $\nu_{D}<1$. This suggests that when the volume $|D|$ is given, the longer the length of $\partial D$ becomes, that is, the more complicated its shape gets, the more $\underline{u}_{\lambda}$ grows up as the diffusion rate $d=1 / \lambda$ goes to infinity. This is due to the acceleration of the inward flux of population owing to the nonlinear effect on $\partial D$, essentially caused by the diffusion rate becoming high. More specifically, letting $D$ be a disc with radius $r$, we may have that the smaller $r$ becomes, the larger $\underline{u}_{\lambda}$ becomes when $\lambda \downarrow 0$.

Finally, we study the existence of large positive solutions $\left(\lambda, u_{\lambda}\right)$ of (1.8) which become unbounded as $\lambda \downarrow 0$, distinguished both from the bifurcation positive solutions to the right at $(\lambda, u)=(0,0)$ and from the secondary bifurcation positive solutions as stated above. If $\int_{D} m \mathrm{~d} x=0$ and $|D|>\int_{\partial D} b \mathrm{~d} \sigma$, then Theorem 1.1 tells us (see (1.6)) that problem (1.8) with $p=2$ admits a positive solution $\left(\lambda, w_{\lambda}\right)$ for $0<\lambda \ll 1$ and $w_{\lambda} \rightarrow 0$ in $C(\bar{D})$ as $\lambda \downarrow 0$. Moreover, there is no secondary bifurcation point for the problem from Theorem 1.6(III). 


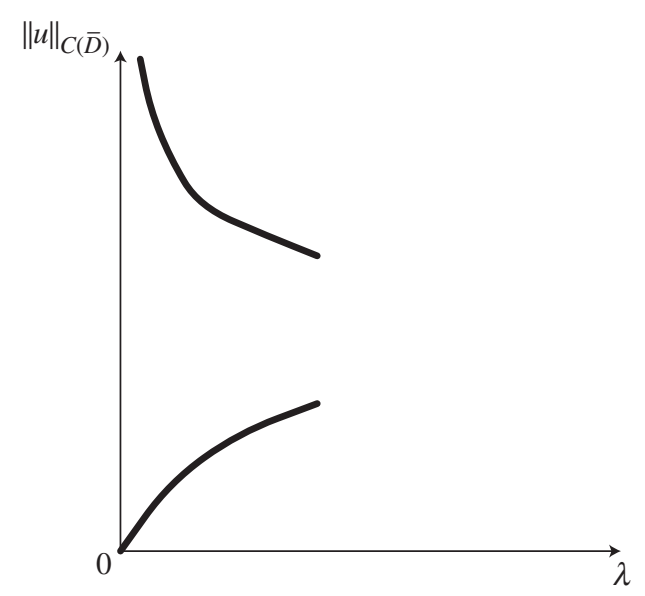

Figure 1. Bifurcations to the right and from infinity at $\lambda=0$.

Now, the following result asserts that problem (1.8) has a second positive solution which grows up to infinity as $\lambda \downarrow 0$.

Theorem 1.8. Let $N=2$ or 3 . Assume $\int_{D} m \mathrm{~d} x=0$. If $|D|>\int_{\partial D} b \mathrm{~d} \sigma$, then problem (1.8) with $p=2$ has a positive solution $\left(\lambda, u_{\lambda}\right)$ for $0<\lambda \ll 1$ such that $u_{\lambda}>w_{\lambda}$, where $w_{\lambda}$ is the first positive solution, as above. Moreover, we have

$$
u_{\lambda} \rightarrow \infty \quad \text { in } C(\bar{D}) \text { as } \lambda \downarrow 0
$$

(see figure 1).

Theorem 1.8 is inspired by Ambrosetti, Brezis and Cerami [4], who consider some semilinear Dirichlet problems with a combined type of concave and convex nonlinearities, in which, based on the first positive solution obtained by means of the super and subsolution method, the existence of a second positive solution is verified by using the mountain-pass theorem. Meanwhile, we prove Theorem 1.8 by considering the corresponding constrained minimization problem.

The case $\int_{D} m \mathrm{~d} x=0$ implies that the operator $-\Delta-\lambda m(x)$ is non-coercive, that is, the principal eigenvalue $\gamma_{1}(\lambda)$ of (1.3) is negative. On the other hand, for the coercive case, or the case $\int_{D} m \mathrm{~d} x<0$ (see [1]), problem (1.8) has at least one positive solution for any $0<\lambda<\lambda_{1}(m)$ by using a variational technique whenever $2 \leqslant p<N /(N-2)$ and $N=2,3$ (see [11, Theorem 3]). In particular, if $p=2$ and $|D| \geqslant \int_{\partial D} b \mathrm{~d} \sigma$, then problem (1.8) has neither bifurcation to the right at $(\lambda, u)=(0,0)$ due to the local bifurcation theory, nor secondary bifurcation from Theorem 1.6 (II). Consequently, the positive solution $\left(\lambda, u_{\lambda}\right)$ constructed by Pflüger will satisfy (1.9) in this case.

Section 2 is devoted to the Lyapunov and Schmidt reduction of (1.1) to a bifurcation equation, in preparation for the proofs of Theorems 1.1 and 1.6. In $\S 3$ we prove Theorem 1.1 and Corollary 1.3. Theorems 1.6 and 1.8 are proved in $\S \S 4$ and 5, respectively. 


\section{Reduction}

In this section we assume that nonlinearity $g$ is sufficiently smooth on $[0, \infty)$. The standard argument provides us with the orthogonal decomposition $L^{2}(D)=\mathbb{R} \oplus V$ of the usual Lebesgue space, where

$$
V=\left\{v \in L^{2}(D): \int_{D} v \mathrm{~d} x=0\right\}
$$

and the projection $Q: L^{2}(D) \rightarrow V$ is of the form

$$
v=Q u:=u-\frac{1}{|D|} \int_{D} u \mathrm{~d} x .
$$

If $u$ is a solution of (1.1), then we have, by means of $Q$,

$$
\left.\begin{array}{cc}
-\Delta v+\frac{\lambda}{|D|} \int_{\partial D} b g(\alpha+v) \mathrm{d} \sigma=\lambda Q f(x, \alpha+v) & \text { in } D, \\
\frac{\partial v}{\partial \boldsymbol{n}}=\lambda b g(\alpha+v) & \text { on } \partial D,
\end{array}\right\}
$$

where $u=\alpha+v \in \mathbb{R} \oplus V$ and $f(x, u)=m(x) u-u^{2}$.

To solve (2.1) in the Hölder space $C^{2+\theta}(\bar{D})$, we put

$$
X=\left\{v \in C^{2+\theta}(\bar{D}): \int_{D} v \mathrm{~d} x=0\right\}
$$

and introduce a nonlinear mapping $F: \mathbb{R} \times \mathbb{R} \times X \rightarrow Z$ as

$$
F(\lambda, \alpha, v)=\left(-\Delta v+\frac{\lambda}{|D|} \int_{\partial D} b g(\alpha+v) \mathrm{d} \sigma-\lambda Q f(x, \alpha+v), \frac{\partial v}{\partial \boldsymbol{n}}-\lambda b g(\alpha+v)\right),
$$

where

$$
Z=\left\{(\phi, \psi) \in C^{\theta}(\bar{D}) \times C^{1+\theta}(\partial D): \int_{D} \phi \mathrm{d} x+\int_{\partial D} \psi \mathrm{d} \sigma=0\right\} .
$$

By condition (1.2) we have the Fréchet derivative $F_{v}(0, c, 0) v=(-\Delta v, \partial v / \partial \boldsymbol{n})$ of $F$ at $(\lambda, \alpha, v)=(0, c, 0)$, where $c \geqslant 0$ is a constant. Banach's closed-graph theorem permits $F_{v}(0, c, 0)$ to be isomorphic. By virtue of the implicit function theorem, the set $F(\lambda, \alpha, v)=0$ consists of a $C^{1}$-function $v=v(\lambda, \alpha)$ in a neighbourhood of $(\lambda, \alpha)=(0, c)$, satisfying $v(0, c)=0$. By the substitution of $v(\lambda, \alpha)$ for $(2.2)$ we obtain the bifurcation equation

$$
\lambda\left(\int_{D} f(x, \alpha+v(\lambda, \alpha)) \mathrm{d} x+\int_{\partial D} b g(\alpha+v(\lambda, \alpha)) \mathrm{d} \sigma\right)=0 .
$$

To sum up, in order to consider the set of non-trivial solutions of $(1.1)$ near $(\lambda, u)=(0, c)$, it suffices to study the equation

$$
\Phi(\lambda, \alpha):=\int_{D} f(x, \alpha+v(\lambda, \alpha)) \mathrm{d} x+\int_{\partial D} b g(\alpha+v(\lambda, \alpha)) \mathrm{d} \sigma=0
$$

near $(\lambda, \alpha)=(0, c)$. 


\section{Bifurcation to the right}

Proof of Theorem 1.1. This section is devoted to the proofs of Theorem 1.1 and Corollary 1.3. First we prove Theorem 1.1, where we need (2.3) with $c=0$. It should be remarked that since $g$ is analytic at $u=0$, the function $v$ is also analytic at $(\lambda, \alpha)=(0,0)$ by the regularity argument on the implicit function theorem (see [13]). It follows that $\Phi$ is also analytic at $(\lambda, \alpha)=(0,0)$.

Lemma 3.1. Let $v=v(\lambda, \alpha)$ be the analytic function in some neighbourhood of $(\lambda, \alpha)=(0,0)$, as given in the case $c=0$ in $\S 2$. Then we have

$$
v(\lambda, 0)=0 \quad \text { for all } \lambda \text { close to } 0,
$$

and the following partial derivatives of $v$ are obtained:

$$
\begin{array}{ll}
\frac{\partial^{j} v}{\partial \lambda^{j}}(0,0) & =0 \quad \text { for all } j \geqslant 1, \\
\frac{\partial^{j} v}{\partial \alpha^{j}}(0,0) & =0 \quad \text { for all } j \geqslant 1, \\
v_{\lambda \alpha}(0,0) & =w_{1},
\end{array}
$$

where $w_{1}$ is a unique solution, in $X$, of the Neumann problem

$$
\begin{array}{rlrl}
-\Delta w & =m & & \text { in } D, \\
\frac{\partial w}{\partial \boldsymbol{n}}=0 & & \text { on } \partial D .
\end{array}
$$

Proof. The proof is due to simple and direct calculations. As seen above, problem (2.1) is uniquely solvable near $(\lambda, \alpha, v)=(0,0,0)$ by $v=v(\lambda, \alpha)$. Problem $(2.1)$ with $(\lambda, \alpha)=$ $(\lambda, 0)$ is

$$
\left.\begin{array}{rlrl}
-\Delta v(\lambda, 0)+\frac{\lambda}{|D|} \int_{\partial D} b g(v(\lambda, 0)) \mathrm{d} \sigma & =\lambda Q f(x, v(\lambda, 0)) & & \text { in } D, \\
\frac{\partial v(\lambda, 0)}{\partial \boldsymbol{n}} & =\lambda b g(v(\lambda, 0)) & & \text { on } \partial D .
\end{array}\right\}
$$

Since $g(0)=0,(3.5)$ admits $v(\lambda, 0)=0$. Hence assertion (3.1) has been verified. Assertion (3.2) is straightforward from (3.1). Assertion (3.3) is clear from (2.1).

Finally, we verify (3.4). Differentiate (2.1) with respect to $\lambda$ and then $\alpha$, and we obtain

$$
\begin{array}{rr}
-\Delta v_{\lambda \alpha}=- & \frac{1}{|D|} \int_{\partial D} b\left\{g^{\prime}(\alpha+v)\left(1+v_{\alpha}\right)+\lambda\left(g^{\prime \prime}(\alpha+v)\left(1+v_{\alpha}\right) v_{\lambda}+g^{\prime}(\alpha+v) v_{\lambda \alpha}\right)\right\} \mathrm{d} \sigma \\
& +Q\left[f_{u}(x, \alpha+v)\left(1+v_{\lambda}\right)+\lambda\left(f_{u u}(x, \alpha+v)\left(1+v_{\alpha}\right) v_{\lambda}+f_{u}(x, \alpha+v) v_{\lambda \alpha}\right)\right] \\
\frac{\partial v_{\lambda \alpha}}{\partial \boldsymbol{n}}=b\left\{g^{\prime}(\alpha+v)\left(1+v_{\alpha}\right)+\lambda\left(g^{\prime \prime}(\alpha+v) v_{\lambda}+g^{\prime}(\alpha+v) v_{\lambda \alpha}\right)\right\} & \text { in } D,
\end{array}
$$


Since $g^{\prime}(0)=0, v_{\lambda}(0,0)=0$ from $(3.2), f_{u}(x, 0)=m$, and $Q m=m$ from $\int_{D} m \mathrm{~d} x=0$, we see that

$$
\begin{array}{rlrl}
-\Delta v_{\lambda \alpha}(0,0) & =m & & \text { in } D, \\
\frac{\partial v_{\lambda \alpha}(0,0)}{\partial \boldsymbol{n}}=0 & & \text { on } \partial D .
\end{array}
$$

From the fact that $v_{\lambda \alpha}(0,0) \in X$, it follows that $v_{\lambda \alpha}(0,0)=w_{1}$. The proof of this lemma is complete.

From (3.2) we note that the solution $u(\lambda, \alpha)=\alpha+v(\lambda, \alpha)$ associated with the zero $(\lambda, \alpha)$ of $\Phi$ near $(\lambda, \alpha)=(0,0)$ is positive if and only if $\alpha>0$. Indeed, by the analyticity of $v$, condition (3.2) gives

$$
\begin{aligned}
u(\lambda, \alpha) & =\alpha+v(\lambda, \alpha) \\
& =\alpha(1+\text { terms of order } k \geqslant 1) \quad \text { as }(\lambda, \alpha) \rightarrow(0,0),
\end{aligned}
$$

as desired. As a result of this fact we have the following proposition.

Proposition 3.2. $(\lambda, u)=(0,0)$ is a bifurcation point to the right for (1.1) if and only if $(\lambda, \alpha)=(0,0)$ for $\Phi$ is as well.

From Lemma 3.1 we can derive

$$
\left.\begin{array}{c}
\Phi_{\lambda}(0,0)=\Phi_{\alpha}(0,0)=\Phi_{\lambda \lambda}(0,0)=0 \\
\Phi_{\lambda \alpha}(0,0)=\int_{D}\left|\nabla w_{1}\right|^{2} \mathrm{~d} x, \\
\Phi_{\alpha \alpha}(0,0)=-2|D|+g^{\prime \prime}(0) \int_{\partial D} b \mathrm{~d} \sigma .
\end{array}\right\}
$$

This is due to the direct computations

$$
\begin{gathered}
\Phi_{\lambda}=\int_{D} f_{u}(x, \alpha+v) v_{\lambda} \mathrm{d} x+\int_{\partial D} b g^{\prime}(\alpha+v) v_{\lambda} \mathrm{d} \sigma \\
\Phi_{\alpha}=\int_{D} f_{u}(x, \alpha+v)\left(1+v_{\alpha}\right) \mathrm{d} x+\int_{\partial D} b g^{\prime}(\alpha+v)\left(1+v_{\alpha}\right) \mathrm{d} \sigma \\
\Phi_{\lambda \lambda}=\int_{D}\left\{f_{u u}(x, \alpha+v)\left(v_{\lambda}\right)^{2}+f_{u}(x, \alpha+v) v_{\lambda \lambda}\right\} \mathrm{d} x \\
+\int_{\partial D} b\left\{g^{\prime \prime}(\alpha+v)\left(v_{\lambda}\right)^{2}+g^{\prime}(\alpha+v) v_{\lambda \lambda}\right\} \mathrm{d} \sigma \\
\Phi_{\lambda \alpha}=\int_{D}\left\{f_{u u}(x, \alpha+v)\left(1+v_{\alpha}\right) v_{\lambda}+f_{u}(x, \alpha+v) v_{\lambda \alpha}\right\} \mathrm{d} x \\
+\int_{\partial D} b\left\{g^{\prime \prime}(\alpha+v)\left(1+v_{\alpha}\right) v_{\lambda}+g^{\prime}(\alpha+v) v_{\lambda \alpha}\right\} \mathrm{d} \sigma, \\
\Phi_{\alpha \alpha}=\int_{D}\left\{f_{u u}(x, \alpha+v)\left(1+v_{\alpha}\right)^{2}+f_{u}(x, \alpha+v) v_{\alpha \alpha}\right\} \mathrm{d} x \\
+\int_{\partial D} b\left\{g^{\prime \prime}(\alpha+v)\left(1+v_{\alpha}\right)^{2}+g^{\prime}(\alpha+v) v_{\alpha \alpha}\right\} \mathrm{d} \sigma .
\end{gathered}
$$


From (3.6) we derive the Taylor expansion of $\Phi$ at $(\lambda, \alpha)=(0,0)$ as

$$
\Phi(\lambda, \alpha)=\lambda \alpha \int_{D}\left|\nabla w_{1}\right|^{2} \mathrm{~d} x+\frac{1}{2} \alpha^{2}\left(-2|D|+g^{\prime \prime}(0) \int_{\partial D} b \mathrm{~d} \sigma\right)+\text { terms of order } k,
$$

where $k \geqslant 3$ and $w_{1}$ is not a constant, that is, $\int_{D}\left|\nabla w_{1}\right|^{2} \mathrm{~d} x>0$. This implies that the Morse Lemma (cf. [10, Theorem 3.1.1]) can be applied to (3.7). Eventually, if (1.4) is satisfied, then the bifurcation to the right for $\Phi$ occurs at $(\lambda, \alpha)=(0,0)$, whereas there is no zero $(\lambda, \alpha), \lambda>0$, of $\Phi$ near $(\lambda, \alpha)=(0,0)$ if $g^{\prime \prime}(0)>(2|D|) / \int_{\partial D} b \mathrm{~d} \sigma$.

In the critical case (1.5) it follows from (3.7) that

$$
\Phi(\lambda, \alpha)=\lambda \alpha \int_{D}\left|\nabla w_{1}\right|^{2} \mathrm{~d} x+\text { terms of order } k,
$$

where $k \geqslant 3$. By direct calculations we derive from (3.2) that

$$
\frac{\partial^{j} \Phi}{\partial \lambda^{j}}(0,0)=0 \quad \text { for all } j \geqslant 1
$$

Hence, by the analyticity of $\Phi$, assertion (3.8) implies that $\Phi(\lambda, \alpha)=\alpha \Phi_{1}(\lambda, \alpha),(\lambda, \alpha) \rightarrow$ $(0,0)$, with

$$
\Phi_{1}(\lambda, \alpha)=\lambda \int_{D}\left|\nabla w_{1}\right|^{2} \mathrm{~d} x+\text { terms of order } k,
$$

where $k \geqslant 2$. Since $\int_{D}\left|\nabla w_{1}\right|^{2} \mathrm{~d} x>0$, the implicit function theorem gives us that the set $\Phi_{1}(\lambda, \alpha)=0$ consists of an analytic function $\lambda=\lambda(\alpha)$ near $\alpha=0$ such that $\lambda(0)=0$, $\lambda^{\prime}(0)=0$ and

$$
\lambda^{\prime \prime}(0)=-\frac{\left(\Phi_{1}\right)_{\alpha \alpha}(0,0)}{\left(\Phi_{1}\right)_{\lambda}(0,0)}=-\frac{g^{\prime \prime \prime}(0) \int_{\partial D} b \mathrm{~d} \sigma}{3 \int_{D}\left|\nabla w_{1}\right|^{2} \mathrm{~d} x} .
$$

If $g^{\prime \prime \prime}(0)<0$, then the bifurcation to the right for $\Phi$ occurs at $(\lambda, \alpha)=(0,0)$, whereas there is no zero $(\lambda, \alpha), \lambda>0$, of $\Phi$ near $(\lambda, \alpha)=(0,0)$ if $g^{\prime \prime \prime}(0)>0$.

In the same way we can verify that if $g$ satisfies (1.5) with $g^{\prime \prime \prime}(0)=0$, namely, if 0 is a zero of $g(u)-\left(|D| / \int_{\partial D} b \mathrm{~d} \sigma\right) u^{2}$ of order $k_{0} \geqslant 4$, then the bifurcation to the right for $\Phi$ occurs at $(\lambda, \alpha)=(0,0)$ when $g^{\left(k_{0}\right)}(0)<0$, whereas there is no zero $(\lambda, \alpha), \lambda>0$, of $\Phi$ near $(\lambda, \alpha)=(0,0)$ when $g^{\left(k_{0}\right)}(0)>0$.

Now it remains to verify the case $g(u)=\left(|D| / \int_{\partial D} b \mathrm{~d} \sigma\right) u^{2}$. Direct computations show that all the terms of $\alpha^{j}, j \geqslant 1$, in the Taylor expansion of $\Phi$ do vanish, which is a result of the fact that $g^{(k)}(0)=0$ for all $j \geqslant 3$. Hence we derive from (3.9) that

$$
\Phi_{1}(\lambda, \alpha)=\lambda\left(\int_{D}\left|\nabla w_{1}\right|^{2} \mathrm{~d} x+\text { terms of order } k\right),
$$

where $k \geqslant 1$. This implies that there is no zero $(\lambda, \alpha), \lambda>0$, of $\Phi$ near $(\lambda, \alpha)=(0,0)$. In view of Proposition 3.2, the proof of Theorem 1.1 is complete.

Proof of Corollary 1.3. Next we prove Corollary 1.3. Our argument is based on the method of super and subsolutions. First of all, we should remark that it is possible to construct any positive subsolution of (1.1) sufficiently small in the following manner. 
Lemma 3.3. Let $\varphi_{1}$ be a positive principal eigenfunction of (1.3), corresponding to the principal eigenvalue $\gamma_{1}(\lambda)$. If $g$ is non-negative for $0<u \ll 1$, then there exists a constant $\varepsilon_{0}=\varepsilon_{0}(\lambda)>0$ such that $\varepsilon \varphi_{1}$ is a subsolution of (1.1) for any $0<\varepsilon \leqslant \varepsilon_{0}$.

If $\lim \sup _{u \downarrow 0} g(u) / u^{2}<|D| / \int_{\partial D} b \mathrm{~d} \sigma$, see (1.6), then there exist constants $u_{1}, \delta_{1}>0$ small, such that

$$
g(u) \leqslant\left(\frac{|D|}{\int_{\partial D} b \mathrm{~d} \sigma}-\delta_{1}\right) u^{2}, \quad 0 \leqslant u \leqslant u_{1}
$$

Set

$$
g_{1}(u)=\left(\frac{|D|}{\int_{\partial D} b \mathrm{~d} \sigma}-\delta_{1}\right) u^{2},
$$

and then $g_{1}$ is analytic at $u=0$ and satisfies (1.4). As seen above, the problem

$$
\left.\begin{array}{rlrl}
-\Delta w & =\lambda\left(m w-w^{2}\right) & & \text { in } D, \\
\frac{\partial w}{\partial \boldsymbol{n}} & =\lambda b g_{1}(w) & & \text { on } \partial D,
\end{array}\right\}
$$

has a bifurcation point to the right at $(\lambda, u)=(0,0)$, meaning that problem (3.11) has a positive solution $\left(\lambda, w_{\lambda}\right)$ for $0<\lambda \ll 1$, satisfying

$$
w_{\lambda} \rightarrow 0 \quad \text { in } C(\bar{D}) \text { as } \lambda \downarrow 0 .
$$

Thanks to $(3.10), w_{\lambda}$ is a supersolution of the original problem (1.1).

Since $w_{\lambda}>0$ in $\bar{D}$, Lemma 3.3 allows us to choose some constant $\varepsilon>0$ such that $\varepsilon \varphi_{1} \leqslant w_{\lambda}$ in $\bar{D}$ and $\varepsilon \varphi_{1}$ is a subsolution of (1.1). The super and subsolution method provides us with a positive solution $\left(\lambda, u_{\lambda}\right)$ of $(1.1)$ such that $\varepsilon \varphi_{1} \leqslant u_{\lambda} \leqslant w_{\lambda}$ in $\bar{D}$. Condition (3.12) gives us the desired conclusion.

If $0<\liminf _{u \downarrow 0} h(u) / u^{k} \leqslant \infty$ with an integer $k \geqslant 3$, see (1.7), then there exist constants $\delta_{2}, u_{2}>0$ such that

$$
\delta_{2} u^{k} \leqslant h(u), \quad 0 \leqslant u \leqslant u_{2}
$$

It follows that

$$
g(u) \geqslant \frac{|D|}{\int_{\partial D} b \mathrm{~d} \sigma} u^{2}+\delta_{2} u^{k}=: g_{2}(u), \quad 0 \leqslant u \leqslant u_{2}
$$

and $g_{2}$ is analytic at $u=0$. We consider

$$
\left.\begin{array}{rlrl}
-\Delta w & =\lambda\left(m w-w^{2}\right) & & \text { in } D, \\
\frac{\partial w}{\partial \boldsymbol{n}} & =\lambda b g_{2}(w) & & \text { on } \partial D,
\end{array}\right\}
$$

and Theorem 1.1 tells us that there is no positive solution $(\lambda, u), \lambda>0$, of (3.13) near $(\lambda, u)=(0,0)$, since $\delta_{2}$ is positive.

Now we get the conclusion by arguing by contradiction. Without loss of generality, we may assume to the contrary that the original problem (1.1) has a positive solution 
$\left(\lambda, u_{\lambda}\right)$ for $0<\lambda \ll 1$ satisfying $u_{\lambda} \rightarrow 0$ in $C(\bar{D})$ as $\lambda \downarrow 0$. However, $u_{\lambda}$ is a supersolution of (3.13) and we can construct a subsolution $\varepsilon \varphi_{1}$ of (3.13) such that $\varepsilon \varphi_{1} \leqslant u_{\lambda}$ in $\bar{D}$ from Lemma 3.3. The super and subsolution method allows us to have a positive solution $\left(\lambda, w_{\lambda}\right)$ of $(3.13)$ satisfying $\varepsilon \varphi_{1} \leqslant w_{\lambda} \leqslant u_{\lambda}$ in $\bar{D}$, and thus $w_{\lambda} \rightarrow 0$ in $C(\bar{D})$ as $\lambda \downarrow 0$, a contradiction.

The remaining assertions of Corollary 1.3 can be verified in the same way. The proof of Corollary 1.3 is thus complete.

\section{Secondary bifurcation}

Proof of Theorem 1.6. In this section we prove Theorem 1.6. First we consider the case $\int_{D} m \mathrm{~d} x>0$. However, the case $\int_{D} m \mathrm{~d} x<0$ is quite similar, so that it will be omitted. A necessary condition for $(0, c)$, where $c>0$ is a constant, to be a secondary bifurcation point for (1.8) is that

$$
\phi(c):=\int_{D} m \mathrm{~d} x-c|D|+c^{p-1} \int_{\partial D} b \mathrm{~d} \sigma=0 .
$$

Indeed, let $\left\{\left(\lambda_{j}, u_{\lambda_{j}}\right)\right\}$ be a sequence of positive solutions of (1.8) such that $\lambda_{j} \downarrow 0$ and $u_{\lambda_{j}} \rightarrow c$ in $C(\bar{D})$ as $j \rightarrow \infty$. Then we have, by Green's formula,

$$
\int_{D}\left(m u_{\lambda_{j}}-\left(u_{\lambda_{j}}\right)^{2}\right) \mathrm{d} x+\int_{\partial D} b\left(u_{\lambda_{j}}\right)^{p} \mathrm{~d} \sigma=0 .
$$

When $j \rightarrow \infty$, assertion (4.1) follows.

If $1<p<2$, then $\phi$ has a unique zero $c_{1}>0$, which implies $\Phi\left(0, c_{1}\right)=0$ from (2.3). We see that $\phi^{\prime}\left(c^{*}\right)=0$ if and only if

$$
\left(c^{*}\right)^{p-2}=\frac{|D|}{(p-1) \int_{\partial D} b \mathrm{~d} \sigma},
$$

where $c^{*}<c_{1}$. The condition (4.2) and the fact that $\phi\left(c_{1}\right)=0$ imply that

$$
\begin{aligned}
\frac{\partial \Phi}{\partial \alpha}\left(0, c_{1}\right) & =\int_{D} m \mathrm{~d} x-2 c_{1}|D|+p\left(c_{1}\right)^{p-1} \int_{\partial D} b \mathrm{~d} \sigma \\
& =c_{1}\left\{(p-1)\left(c_{1}\right)^{p-2} \int_{\partial D} b \mathrm{~d} \sigma-|D|\right\} \\
& <c_{1}\left\{(p-1)\left(c^{*}\right)^{p-2} \int_{\partial D} b \mathrm{~d} \sigma-|D|\right\} \\
& =0 .
\end{aligned}
$$

Hence the implicit function theorem provides us with a $C^{1}$-function $\alpha=\alpha(\lambda)$ such that $\alpha(0)=c_{1}$ and

$$
\Phi(\lambda, \alpha)=0 \quad \Longleftrightarrow \quad \alpha=\alpha(\lambda), \quad \text { near }\left(0, c_{1}\right),
$$

as desired. 
If $p=2$ and $|D|>\int_{\partial D} b \mathrm{~d} \sigma$, then the following constant $c_{1}$ uniquely solves the equation $\phi(c)=0$ :

$$
c=\frac{\int_{D} m \mathrm{~d} x}{|D|-\int_{\partial D} b \mathrm{~d} \sigma} \quad\left(\text { denoted by } c_{1}\right) .
$$

It follows that $\Phi\left(0, c_{1}\right)=0$, and that

$$
\frac{\partial \Phi}{\partial \alpha}\left(0, c_{1}\right)=\int_{D} m \mathrm{~d} x-2 c_{1}|D|+2 c_{1} \int_{\partial D} b \mathrm{~d} \sigma=-\int_{D} m \mathrm{~d} x<0 .
$$

Again, using the implicit function theorem leads us to the desired conclusion.

If $p=2$ and $|D| \leqslant \int_{\partial D} b \mathrm{~d} \sigma$, then we note that $\phi(c)>0$ for all $c>0$, so that the secondary bifurcation cannot occur at any $(0, c)$.

If $p>2$, then $\phi^{\prime}$ has a unique zero $c^{*}>0$ given by (4.2), so that

$$
\begin{aligned}
\phi\left(c^{*}\right) & =\int_{D} m \mathrm{~d} x-c^{*}|D|+\left(c^{*}\right)^{p-1} \int_{\partial D} b \mathrm{~d} \sigma \\
& =\int_{D} m \mathrm{~d} x-c^{*}\left(\frac{p-2}{p-1}\right)|D| .
\end{aligned}
$$

Additionally, if $|D|<m_{p} \int_{\partial D} b \mathrm{~d} \sigma$, then we have

$$
c^{*}<\left(\frac{p-1}{p-2}\right) \frac{\int_{D} m \mathrm{~d} x}{|D|},
$$

which implies that $\phi\left(c^{*}\right)>0$. Since $\phi$ is strictly convex, it follows that $\phi(c)>0$ for all $c>0$ and the desired conclusion follows.

On the other hand, if, additionally, $|D|>m_{p} \int_{\partial D} b \mathrm{~d} \sigma$, then the function $\phi$ has exactly two zeros $c_{2}>c_{1}>0$, since $\phi\left(c^{*}\right)<0$. This implies that $\Phi\left(0, c_{j}\right)=0$ and

$$
\begin{aligned}
\frac{\partial \Phi}{\partial \alpha}\left(0, c_{j}\right) & =\int_{D} m \mathrm{~d} x-2 c_{j}|D|+p\left(c_{j}\right)^{p-1} \int_{\partial D} b \mathrm{~d} \sigma \\
& =-c_{j}|D|+(p-1)\left(c_{j}\right)^{p-1} \int_{\partial D} b \mathrm{~d} \sigma .
\end{aligned}
$$

Combining (4.2) and the condition $c_{1}<c^{*}<c_{2}$ ensures that $(\partial \Phi / \partial \alpha)\left(0, c_{j}\right) \neq 0$. The implicit function theorem leads us to the desired conclusion. Assertion (I) of Theorem 1.6 has been verified.

Finally, it remains to verify the case $\int_{D} m \mathrm{~d} x=0$. If $p \neq 2$, then $\phi$ has a unique zero $c_{1}>0$ given by

$$
c_{1}=\left(\frac{|D|}{\int_{\partial D} b \mathrm{~d} \sigma}\right)^{1 /(p-2)}
$$

and then $\left(0, c_{1}\right)$ is sure to be a secondary bifurcation point for (1.8) in the same manner as in the case $\int_{D} m \mathrm{~d} x>0$.

If $p=2$, then we find from (4.1) that the condition $|D|=\int_{\partial D} b \mathrm{~d} \sigma$ is necessary for the secondary bifurcation to occur at some $(0, c)$, which completes the proof of assertion (III) of Theorem 1.6.

We have finished the proof of Theorem 1.6. 


\section{Existence of growing-up solutions}

Proof of Theorem 1.8. This section is devoted to the proof of Theorem 1.8. As seen in Theorem 1.1, there exists a positive solution $\left(\lambda, w_{\lambda}\right)$ of (1.8) with $p=2$ for $0<\lambda \ll 1$, satisfying $w_{\lambda} \rightarrow 0$ in $C(\bar{D})$ as $\lambda \downarrow 0$. Let $u=w_{\lambda}+v$. Then $v \in C^{2}(\bar{D})$ satisfies

$$
\left.\begin{array}{rlrl}
-\Delta v & =\lambda\left(\left(m-2 w_{\lambda}\right) v-v^{2}\right) & & \text { in } D, \\
\frac{\partial v}{\partial \boldsymbol{n}} & =\lambda b\left(2 w_{\lambda} v+v^{2}\right) & & \text { on } \partial D,
\end{array}\right\}
$$

if and only if $u$ is a solution of (1.8) with $p=2$. If $N=2,3$, then the embedding $W^{1,2}(D) \subset L^{3}(D)$ of the Sobolev space is compact and the usual trace operator $W^{1,2}(D) \rightarrow L^{3}(\partial D)$ is also compact. We call $\left(\lambda, v_{\lambda}\right) \in(0, \infty) \times W^{1,2}(D)$ a weak solution of (5.1) if we have, for any $\varphi \in W^{1,2}(D)$,

$$
\begin{aligned}
\int_{D} \nabla v_{\lambda} \nabla \varphi \mathrm{d} x-\lambda \int_{D} \tilde{m}_{\lambda} v_{\lambda} \varphi \mathrm{d} x & +\lambda \int_{D}\left(v_{\lambda}\right)^{2} \varphi \mathrm{d} x \\
& -2 \lambda \int_{\partial D} b w_{\lambda} v_{\lambda} \varphi \mathrm{d} \sigma-\lambda \int_{\partial D} b\left(v_{\lambda}\right)^{2} \varphi \mathrm{d} \sigma=0
\end{aligned}
$$

where $\tilde{m}_{\lambda}=m-2 w_{\lambda}$. By the standard $L^{p}$ regularity theory, a weak solution of (5.1) is in $C^{2}(\bar{D})$, that is, a solution in our sense.

To prove Theorem 1.8, it suffices to prove the existence of a positive solution $V_{\lambda}$ of (5.1) for $0<\lambda \ll 1$. Indeed, $u_{\lambda}=w_{\lambda}+V_{\lambda}$ is a positive solution of (1.8) with $p=2$. If we assume on the contrary that $\lambda_{j} \downarrow 0$ and $\left\|u_{\lambda_{j}}\right\|_{C(\bar{D})} \leqslant M$ with some constant $M>0$ as $j \rightarrow \infty$, then Amann's $L^{p}$ regularity theory (see [3, Proposition 3.3]) shows that

$$
\left\|u_{\lambda_{j}}\right\|_{W^{1, p}(D)} \leqslant C_{p}\left(\left\|(-\Delta+1) u_{\lambda_{j}}\right\|_{L^{p}(D)}+\left\|\frac{\partial u_{\lambda_{j}}}{\partial \boldsymbol{n}}\right\|_{L^{p}(\partial D)}\right), \quad 1<p<\infty,
$$

with some constant $C_{p}>0$. This implies that $\left\|u_{\lambda_{j}}\right\|_{W^{1, p}(D)}$ is bounded with respect to $j \geqslant 1$ for any $1<p<\infty$. By the standard $L^{p}$ regularity theory and the AscoliArzelà theorem, it follows that there exist $\hat{u} \in C^{2}(\bar{D})$ and a subsequence of $\left\{\left(\lambda_{j}, u_{\lambda_{j}}\right)\right\}$, again denoted by $\left\{\left(\lambda_{j}, u_{\lambda_{j}}\right)\right\}$, such that $\lambda_{j} \downarrow 0$ and $u_{\lambda_{j}} \rightarrow \hat{u}$ in $C^{2}(\bar{D})$, so that $\hat{u}$ is a non-negative constant. This is a contradiction, since Theorems 1.1 and 1.6 tell us the existence of a unique bifurcation curve to the right at $(\lambda, u)=(0,0)$ and no secondary bifurcation at any $(0, c)$, where $c>0$ is a constant, respectively, for the problem under consideration. Eventually we have $\left\|u_{\lambda}\right\|_{C(\bar{D})} \rightarrow \infty$ as $\lambda \downarrow 0$.

Now, we associate with (5.1) the following constrained minimization problem: for given

$$
J_{\lambda}(v)=\frac{\lambda}{3} \int_{D}|v|^{3} \mathrm{~d} x-\frac{\lambda}{3} \int_{\partial D} b|v|^{3} \mathrm{~d} \sigma, \quad v \in M_{\lambda},
$$

and

$$
M_{\lambda}=\left\{v \in W^{1,2}(D): E_{\lambda}(v):=\frac{1}{2} \int_{D}|\nabla v|^{2} \mathrm{~d} x-\frac{\lambda}{2} \int_{D} \tilde{m}_{\lambda} v^{2} \mathrm{~d} x-\lambda \int_{\partial D} b w_{\lambda} v^{2} \mathrm{~d} \sigma \leqslant 1\right\},
$$


find $v_{\lambda} \in M_{\lambda}$ such that

$$
v_{\lambda} \neq 0 \quad \text { and } \quad J_{\lambda}\left(v_{\lambda}\right)=\inf _{v \in M_{\lambda}} J_{\lambda}(v)
$$

To do this, we first show that

$$
\inf _{v \in M_{\lambda}} J_{\lambda}(v)<0
$$

Indeed, by considering a suitable $C^{1}$-function in $\bar{D}$ whose support is contained in a neighbourhood of $x \in \partial D$ where $b(x)>0$, it is possible to construct $\tilde{v} \in C^{1}(\bar{D})$ such that $J_{\lambda}(\tilde{v})<0$. If a constant $\varepsilon>0$ is small enough, then $J_{\lambda}(\varepsilon \tilde{v})<0$ and $E_{\lambda}(\varepsilon \tilde{v}) \leqslant 1$. Assertion (5.3) has been proved.

Next we have the following lemma.

Lemma 5.1. There exists a constant $\bar{\lambda}>0$ such that if $0<\lambda \leqslant \bar{\lambda}$, then we can take some constant $C(\lambda)>0$ satisfying that, for any $\left\{v_{j}\right\} \subset M_{\lambda}$ being a minimizing sequence for (5.2), that is,

$$
J_{\lambda}\left(v_{j}\right) \downarrow \inf _{v \in M_{\lambda}} J_{\lambda}(v) \in[-\infty, 0),
$$

we have $\left\|v_{j}\right\|_{W^{1,2}(D)} \leqslant C(\lambda)$.

Once Lemma 5.1 is proved, by the standard compactness argument there exist $v_{\lambda} \in$ $W^{1,2}(D), 0<\lambda \leqslant \bar{\lambda}$, and a sub-sequence of $\left\{v_{j}\right\}$, again denoted by $\left\{v_{j}\right\}$, such that

$$
\begin{array}{ll}
v_{j} \rightarrow v_{\lambda} & \text { weakly in } W^{1,2}(D), \\
v_{j} \rightarrow v_{\lambda} & \text { strongly both in } L^{3}(D) \text { and in } L^{3}(\partial D) .
\end{array}
$$

It follows that, as $j \rightarrow \infty$,

$$
J_{\lambda}\left(v_{j}\right) \rightarrow \frac{\lambda}{3} \int_{D}\left|v_{\lambda}\right|^{3} \mathrm{~d} x-\frac{\lambda}{3} \int_{\partial D} b\left|v_{\lambda}\right|^{3} \mathrm{~d} \sigma=J_{\lambda}\left(v_{\lambda}\right)=\inf _{v \in M_{\lambda}} J_{\lambda}(v)>-\infty .
$$

By the lower semicontinuity of $E_{\lambda}(\cdot)$ we have $E_{\lambda}\left(v_{\lambda}\right) \leqslant 1$, as desired. Here it should be noted that the minimizer $v_{\lambda}$ is allowed to be non-negative in $D$ (if not, then $v_{\lambda}$ is replaced by $\left.\left|v_{\lambda}\right|\right)$, and we can in fact show that

$$
E_{\lambda}\left(v_{\lambda}\right)=1
$$

Assume to the contrary that $E_{\lambda}\left(v_{\lambda}\right)<1$, that is, $v_{\lambda}$ is an interior point of $M_{\lambda}$. Then, for any $\varphi \in W^{1,2}(D)$, we obtain $v_{\lambda}+s \varphi \in M_{\lambda},|s| \ll 1$, and

$$
\left.\frac{\mathrm{d}}{\mathrm{d} s} J_{\lambda}\left(v_{\lambda}+s \varphi\right)\right|_{s=0}=0
$$

On the other hand, by direct computations we have

$$
\left.\frac{\mathrm{d}}{\mathrm{d} s} J_{\lambda}\left(v_{\lambda}+s \varphi\right)\right|_{s=0}=\lambda\left(\int_{D}\left(v_{\lambda}\right)^{2} \varphi \mathrm{d} x-\int_{\partial D} b\left(v_{\lambda}\right)^{2} \varphi \mathrm{d} \sigma\right) \quad \text { for any } \varphi \in W^{1,2}(D) .
$$


Choose $\varphi$ such that $\varphi=v_{\lambda}$, and we obtain

$$
\left.\frac{\mathrm{d}}{\mathrm{d} s} J_{\lambda}\left(v_{\lambda}+s v_{\lambda}\right)\right|_{s=0}=3 J_{\lambda}\left(v_{\lambda}\right)<0
$$

which contradicts (5.4), as desired. Therefore, we have proved that there exists $v_{\lambda} \in S_{\lambda}$, where

$$
S_{\lambda}=\left\{v \in W^{1,2}(D): E_{\lambda}(v)=1\right\},
$$

such that $v_{\lambda} \geqslant 0, v_{\lambda} \neq 0$ and

$$
J_{\lambda}\left(v_{\lambda}\right)=\inf _{v \in S_{\lambda}} J_{\lambda}(v)<0 .
$$

Now, it is straightforward to prove that the minimizer $v_{\lambda}$ satisfies

$$
J_{\lambda}^{\prime}\left(v_{\lambda}\right) \varphi+\Lambda E_{\lambda}^{\prime}\left(v_{\lambda}\right) \varphi=0 \quad \text { for any } \varphi \in W^{1,2}(D),
$$

where $\Lambda$ is the corresponding Lagrange multiplier. Putting $\varphi=v_{\lambda}$, we have $\Lambda=$ $-3 J_{\lambda}\left(v_{\lambda}\right) / 2>0$. Set $V_{\lambda}=\Lambda^{-1} v_{\lambda}$, and then $\left(\lambda, V_{\lambda}\right)$ is a weak solution of (5.1) that is non-negative and non-zero. Hence $V_{\lambda} \in C^{2}(\bar{D})$ as already stated. By the strong maximum principle and the boundary point lemma it follows that $V_{\lambda}>0$ in $\bar{D}$, as desired.

For the completeness of the proof it remains to prove Lemma 5.1.

Proof of Lemma 5.1. For the orthogonal decomposition $W^{1,2}(D)=\mathbb{R} \oplus W$, where $W=\left\{\eta \in W^{1,2}(D): \int_{D} \eta \mathrm{d} x=0\right\}$, we denote by $\|\cdot\|_{W}$ a reduced norm to $W$ in $W^{1,2}(D)$. It is easy to see that $\|v\|_{W^{1,2}(D)}^{2}$ and $|t|^{2}+\|\eta\|_{W}^{2}$ are equivalent for $v=t+\eta \in W^{1,2}(D)$, where $t \in \mathbb{R}$ and $\eta \in W$. We can also check that $\|\eta\|_{W}^{2}$ and $\int_{D}|\nabla \eta|^{2} \mathrm{~d} x$ are equivalent for $\eta \in W$, and that there exist constants $C_{0}>0$ and $\lambda^{*}>0$ such that $C_{0}\|\eta\|_{W}^{2} \leqslant E_{\lambda}(\eta)$ for all $\eta \in W$ and $0<\lambda \leqslant \lambda^{*}$, since $W \subset L^{2}(D), L^{2}(\partial D)$ are both continuous.

First we prove the following proposition.

Proposition 5.2. Let $0<\lambda \leqslant \lambda^{*}$. Then there exists a constant $C_{1}(\lambda)>0$ satisfying $C_{1}(\lambda) \rightarrow 0, \lambda \downarrow 0$, such that the following assertion holds true: for any $\left\{v_{j}\right\} \subset W^{1,2}(D)$ such that

$$
E_{\lambda}\left(v_{j}\right) \leqslant 1
$$

and

$$
\left\|v_{j}\right\|_{W^{1,2}(D)} \rightarrow \infty \quad \text { as } j \rightarrow \infty
$$

we have

$$
\limsup _{j \rightarrow \infty}\left\|\frac{\eta_{j}}{t_{j}}\right\|_{W} \leqslant C_{1}(\lambda)
$$

where $v_{j}=t_{j}+\eta_{j} \in \mathbb{R} \oplus W$. 
Proof. We derive from the condition $E_{\lambda}\left(v_{j}\right) \leqslant 1$ that

$$
\begin{aligned}
C_{0}\left\|\eta_{j}\right\|_{W}^{2} \leqslant 1+\frac{1}{2} \lambda\left(t_{j}\right)^{2} \int_{D} \tilde{m}_{\lambda} \mathrm{d} x & +\lambda t_{j} \int_{D} \tilde{m}_{\lambda} \eta_{j} \mathrm{~d} x \\
& +\lambda\left(t_{j}\right)^{2} \int_{\partial D} b w_{\lambda} \mathrm{d} \sigma+2 \lambda t_{j} \int_{\partial D} b w_{\lambda} \eta_{j} \mathrm{~d} \sigma .
\end{aligned}
$$

If $\left|t_{j}\right|$ is bounded, then condition (5.6) ensures that $\left\|\eta_{j}\right\|_{W} \rightarrow \infty$ as $j \rightarrow \infty$. However, by using Schwarz's inequality, the right-hand side of (5.7) is bounded above for $C_{2}\left(1+\left\|\eta_{j}\right\|_{W}\right)$ with some constant $C_{2}>0$ independent of $j$, which leads to a contradiction when $j \rightarrow \infty$ in (5.7). Hence we have $\left|t_{j}\right| \rightarrow \infty$ as $j \rightarrow \infty$.

Using Schwarz's inequality, we derive from (5.7) that

$$
\begin{aligned}
C_{0}\left\|\frac{\eta_{j}}{t_{j}}\right\|_{W}^{2} & \leqslant \frac{1}{\left(t_{j}\right)^{2}}+\frac{\lambda}{2} \int_{D} \tilde{m}_{\lambda} \mathrm{d} x+\lambda \int_{D} \tilde{m}_{\lambda} \frac{\eta_{j}}{t_{j}} \mathrm{~d} x+\lambda \int_{\partial D} b w_{\lambda} \mathrm{d} \sigma+2 \lambda \int_{\partial D} b w_{\lambda} \frac{\eta_{j}}{t_{j}} \mathrm{~d} \sigma \\
& \leqslant \frac{1}{\left(t_{j}\right)^{2}}+C_{3}(\lambda)+C_{4}(\lambda)\left\|\frac{\eta_{j}}{t_{j}}\right\|_{W}
\end{aligned}
$$

Here $C_{3}(\lambda)$ and $C_{4}(\lambda)$ are positive constants independent of the choice of the $\left\{v_{j}\right\}$, satisfying

$$
\lim _{\lambda \downarrow 0} C_{3}(\lambda)=\lim _{\lambda \downarrow 0} C_{4}(\lambda)=0
$$

Solving (5.8), we obtain

$$
\left\|\frac{\eta_{j}}{t_{j}}\right\|_{W} \leqslant \frac{C_{4}(\lambda)+\sqrt{C_{4}(\lambda)^{2}+4 C_{0}\left\{\left(t_{j}\right)^{-2}+C_{3}(\lambda)\right\}}}{2 C_{0}}
$$

which implies that

$$
\limsup _{j \rightarrow \infty}\left\|\frac{\eta_{j}}{t_{j}}\right\|_{W} \leqslant \frac{C_{4}(\lambda)+\sqrt{C_{4}(\lambda)^{2}+4 C_{0} C_{3}(\lambda)}}{2 C_{0}}=: C_{1}(\lambda) .
$$

We derive the desired conclusion from (5.9), and Proposition 5.2 has been verified.

Since $W \subset L^{3}(D), L^{3}(\partial D)$ are both continuous and $|D|>\int_{\partial D} b \mathrm{~d} \sigma$, we can choose constants $\varepsilon_{0}>0, C_{5}>0$ and $0<\lambda^{* *}<\lambda^{*}$ such that

$$
C_{1}(\lambda)<\frac{1}{2} \varepsilon_{0} \quad \text { if } 0<\lambda \leqslant \lambda^{* *}
$$

and

$$
\int_{D}|1+\eta|^{3} \mathrm{~d} x-\int_{\partial D} b|1+\eta|^{3} \mathrm{~d} \sigma \geqslant C_{5} \quad \text { if } \eta \in W \text { and }\|\eta\|_{W}<\varepsilon_{0} .
$$

Now let us show how to prove Lemma 5.1 with $\bar{\lambda}=\lambda^{* *}$. Assume to the contrary that there exists $0<\lambda \leqslant \lambda^{* *}$ such that some minimizing sequence $\left\{v_{j}\right\} \subset M_{\lambda}$ includes a subsequence satisfying (5.6), again denoted by $\left\{v_{j}\right\}$. Since $\left\{v_{j}\right\}$ satisfies (5.5), Proposition 5.2 
can be applied to the sub-sequence $\left\{v_{j}\right\}$ and we get, from (5.10),

$$
\limsup _{j \rightarrow \infty}\left\|\frac{\eta_{j}}{t_{j}}\right\|_{W} \leqslant \frac{1}{2} \varepsilon_{0}, \quad v_{j}=t_{j}+\eta_{j} \in \mathbb{R} \oplus W .
$$

From (5.11) it follows that there exists $j_{0}(\lambda) \geqslant 1$ such that, for any $j \geqslant j_{0}(\lambda)$,

$$
J_{\lambda}\left(v_{j}\right)=\frac{1}{3} \lambda\left|t_{j}\right|^{3}\left(\int_{D}\left|1+\frac{\eta_{j}}{t_{j}}\right|^{3} \mathrm{~d} x-\int_{\partial D} b\left|1+\frac{\eta_{j}}{t_{j}}\right|^{3} \mathrm{~d} \sigma\right) \geqslant \frac{1}{3} \lambda\left|t_{j}\right|^{3} C_{5},
$$

which contradicts (5.3) when $j \rightarrow \infty$, as desired. The proof of Lemma 5.1 is complete.

The proof of Theorem 1.8 is now complete.

Acknowledgements. The author thanks the referee for careful reading of the manuscript and some valuable comments and suggestions.

\section{References}

1. G. A. Afrouzi And K. J. Brown, On principal eigenvalues for boundary value problems with indefinite weight and Robin boundary conditions, Proc. Am. Math. Soc. 127 (1999), $125-130$.

2. H. Amann, Fixed point equations and nonlinear eigenvalue problems in ordered Banach spaces, SIAM Rev. 18 (1976), 620-709.

3. H. Amann, Nonlinear elliptic equations with nonlinear boundary conditions, in New developments in differential equations (ed. W. Eckhaus), Mathematical Studies, vol. 21, pp. 4363 (Amsterdam, North-Holland, 1976).

4. A. Ambrosetti, H. Brezis and G. Cerami, Combined effects of concave and convex nonlinearities in some elliptic problems, J. Funct. Analysis 122 (1994), 519-543.

5. R. S. Cantrell and C. Cosner, Diffusive logistic equations with indefinite weights: population models in disrupted environments, Proc. R. Soc. Edinb. A 112 (1989), 293318.

6. R. S. Cantrell and C. Cosner, Diffusive logistic equations with indefinite weights: population models in disrupted environments, II, SIAM J. Math. Analysis 22 (1991), 1043-1064.

7. M. G. Crandall and P. H. Rabinowitz, Bifurcation from simple eigenvalues, J. Funct. Analysis 8 (1971), 321-340.

8. P. Hess, Periodic-parabolic boundary value problems and positivity, Pitman Research Notes in Mathematics Series, vol. 247 (Harlow, Essex, Longman Scientific and Technical, 1991).

9. J. LÓPEZ-GómEz, Spectral theory and nonlinear functional analysis, Chapman and Hall/CRC Research Notes in Mathematics, vol. 426 (Boca Raton, FL, Chapman and Hall/CRC, 2001).

10. L. NiRENBERG, Topics in nonlinear functional analysis, Courant Lecture Notes in Mathematics, vol. 6 (Providence, RI, American Mathematical Society, 2001).

11. K. Pflüger, On indefinite nonlinear problems, in Partial differential and integral equations (ed. H. G. W. Begehr et al.), pp. 335-346 (Kluwer, 1999).

12. M. H. Protter ANd H. F. WeinBerger, Maximum principles in differential equations (Englewood Cliffs NJ, Prentice-Hall, 1967).

13. E. ZEIDLER, Nonlinear functional analysis and its applications, vol. I: fixed-point theorems (Springer, 1993). 